University of New Orleans

ScholarWorks@UNO

8-1989

\title{
Analytical determination of the complex dielectric function of an absorbing medium from two angles of incidence of minimum parallel reflectance
}

R. M.A. Azzam

University of New Orleans, razzam@uno.edu

Follow this and additional works at: https://scholarworks.uno.edu/ee_facpubs

Part of the Electrical and Electronics Commons, and the Optics Commons

\section{Recommended Citation}

R. M. A. Azzam, "Analytical determination of the complex dielectric function of an absorbing medium from two angles of incidence of minimum parallel reflectance," J. Opt. Soc. Am. A 6, 1213-1216 (1989)

This Article is brought to you for free and open access by the Department of Electrical Engineering at ScholarWorks@UNO. It has been accepted for inclusion in Electrical Engineering Faculty Publications by an authorized administrator of ScholarWorks@UNO. For more information, please contact scholarworks@uno.edu. 


\title{
Analytical determination of the complex dielectric function of an absorbing medium from two angles of incidence of minimum parallel reflectance
}

\author{
R. M. A. Azzam \\ Department of Electrical Engineering, University of New Orleans, Lakefront, New Orleans, Louisiana 70148 \\ Received January 10, 1989; accepted April 3, 1989

\begin{abstract}
The real and imaginary parts of the complex dielectric function (or complex refractive index) of an opaque substrate or a thick film can be determined from two pseudo-Brewster angles measured in two transparent incidence media of different refractive indices. This two-angle method is simple in that it involves no photometric or polarimetric analysis and in that the solution for the optical properties in terms of the measured angles is explicit, analytical, and direct (i.e. noniterative). The two-angle method is demonstrated for an opaque TiN film on a Cleartran ZnS substrate as a specific example. The effect of angle-of-incidence errors on the determination of the optical
\end{abstract} \\ properties is investigated, and the domain of applicability of this new and interesting method is also delineated.
}

\section{INTRODUCTION}

Determination of the intrinsic optical properties of materials is important for solid-state physics ${ }^{1}$ and for applications that involve optical coatings. ${ }^{2}$ In the presence of strong absorption, these properties (specifically, the real and imaginary parts of the complex dielectric function $\left.\epsilon=\epsilon_{r}+j \epsilon_{i}\right)$ are determined mainly by use of spectral-reflectance ${ }^{3}$ or spectroscopic-ellipsometry ${ }^{4}$ methods.

In this paper a new and simple method is described for the determination of the complex $\epsilon$ of an optically isotropic (homogeneous, linear, and nonmagnetic) medium that differs from spectral-reflectance and spectroscopic-ellipsometry methods in that it does not require absolute or relative photometric measurement or polarization analysis of the reflected light. The method is based on a generalization of Brewster's law that determines $\epsilon_{r}$ of a transparent medium by $\epsilon_{r}=\tan ^{2} \phi_{\mathrm{B}}$, in which $\phi_{\mathrm{B}}$ is the angle of incidence of minimum (zero) reflectance for incident $p$-polarized light $\left(R_{p}\right)$. For light reflection at the planar surface of an absorbing medium, $R_{p}$ reaches a nonzero minimum at the so-called pseudo-Brewster angle $\phi_{\mathrm{pB}}$, which is a function of $\epsilon_{r}$ and $\epsilon_{i}$. Two pseudo-Brewster angles $\phi_{\mathrm{pB} 1}$ and $\phi_{\mathrm{pB} 2}$, measured with the $p$-polarized light incident from two different transparent media of known refractive indices, provide sufficient information to determine $\epsilon_{r}$ and $\epsilon_{i}$. This nonphotometric, nonpolarimetric, two-angle method (TAM) has the further advantage that the inversion that determines the desired parameters from the measured data is analytical, explicit, and noniterative.

\section{MATHEMATICAL BASIS OF THE TWO-ANGLE METHOD}

The pseudo-Brewster angle is determined in terms of complex $\epsilon$ by solving the following cubic equation:

$$
\left(2 \epsilon_{r}+2|\epsilon|^{2}\right) u^{3}+\left(|\epsilon|^{4}-3|\epsilon|^{2}\right) u^{2}-2|\epsilon|^{4} u+|\epsilon|^{4}=0,
$$

where $u=\sin ^{2} \phi$, and the subscript $\mathrm{pB}$ is dropped for sim- plicity. Equation (1) or equivalent equations have been derived by several authors. ${ }^{5-7}$ To develop the TAM, Eq. (1) is considered as a quadratic equation in $|\epsilon|^{2}$ whose solution can be manipulated to yield

$$
y=\left[a+\left(a^{2}-b x\right)^{1 / 2}-x^{2}\right]^{1 / 2},
$$

in which

$$
\begin{aligned}
& x=\epsilon_{r}, \quad y=\epsilon_{i}, \\
& a=\tan ^{4} \phi\left(1.5-\sin ^{2} \phi\right), \\
& b=2 \tan ^{4} \phi \sin ^{2} \phi .
\end{aligned}
$$

Equation (2) is important in that it gives the explicit Cartesian equation of the equi-pseudo-Brewster-angle contour in the complex $\epsilon$ plane. When $\phi$ is allowed to assume all possible values, a complete family of such curves is generated that fills the entire plane. The properties of these contours will be discussed in a forthcoming paper.

Measuring two pseudo-Brewster angles $\phi_{1}$ and $\phi_{2}$ in two transparent incidence media of different refractive indices $\epsilon_{01}{ }^{1 / 2}$ and $\epsilon_{02}{ }^{1 / 2}$ allows us to write Eq. (2) twice:

$$
\begin{aligned}
& y_{1}=\left[a_{1}+\left(a_{1}{ }^{2}-b_{1} x_{1}\right)^{1 / 2}-x_{1}{ }^{2}\right]^{1 / 2}, \\
& y_{2}=\left[a_{2}+\left(a_{2}{ }^{2}-b_{2} x_{2}\right)^{1 / 2}-x_{2}{ }^{2}\right]^{1 / 2},
\end{aligned}
$$

in which

$$
\begin{aligned}
& x_{1}+j y_{1}=\epsilon / \epsilon_{01}, \\
& x_{2}+j y_{2}=\epsilon / \epsilon_{02} .
\end{aligned}
$$

Because $\epsilon_{01}$ and $\epsilon_{02}$ are real, Eqs. (7) and (8) define two parallel vectors; hence we can write

$$
\begin{gathered}
x_{2}=x_{1} / \epsilon_{0}, \quad y_{2}=y_{1} / \epsilon_{0}, \\
\epsilon_{0}=\epsilon_{02} / \epsilon_{01} .
\end{gathered}
$$

Substitution of Eqs. (9) into Eq. (6) gives

$$
y_{1}=\left[\epsilon_{0}{ }^{2} a_{2}+\left(\epsilon_{0}{ }^{4} a_{2}{ }^{2}-\epsilon_{0}{ }^{3} b_{2} x_{1}\right)^{1 / 2}-x_{1}{ }^{2}\right]^{1 / 2} \text {. }
$$


Equating the squares of the right-hand sides of Eqs. (5) and (11), we obtain, after considerable simplification,

$$
x_{1}=\left(4 \epsilon_{0}^{2}\right) \frac{\left(\epsilon_{0}^{2} a_{2}-a_{1}\right)\left(\epsilon_{0} a_{1} b_{2}-a_{2} b_{1}\right)}{\left(b_{1}-\epsilon_{0}^{3} b_{2}\right)^{2}} .
$$

Equation (12) gives an explicit analytical solution for the real part of the complex dielectric function $\epsilon / \epsilon_{01}$ in terms of $\phi_{1}, \phi_{2}$, and $\epsilon_{0}$. The imaginary part, $y_{1}$, is determined subsequently by direct substitution into the right-hand side of Eq. (5). $\epsilon$ is determined next by

$$
\epsilon=\epsilon_{01}\left(x_{1}+j y_{1}\right) .
$$

This completes the mathematical development of the TAM.

\section{BULK AND THICK-FILM SAMPLES FOR THE TWO-ANGLE METHOD}

To apply the TAM, the absorbing sample may have one planar surface available for specular reflection measurements. For this case of a bulk liquid or solid sample, the first pseudo-Brewster angle $\phi_{1}$ may be measured with the light incident from vacuum, air, or an inert gas, so that $\epsilon_{01}=$ 1. For $\phi_{2}$, a transparent and dense medium of incidence is required whose dielectric function $\epsilon_{02}$ is known or is determined separately. (It is also possible, and may even be desirable, to use two different dense media of incidence, e.g.,
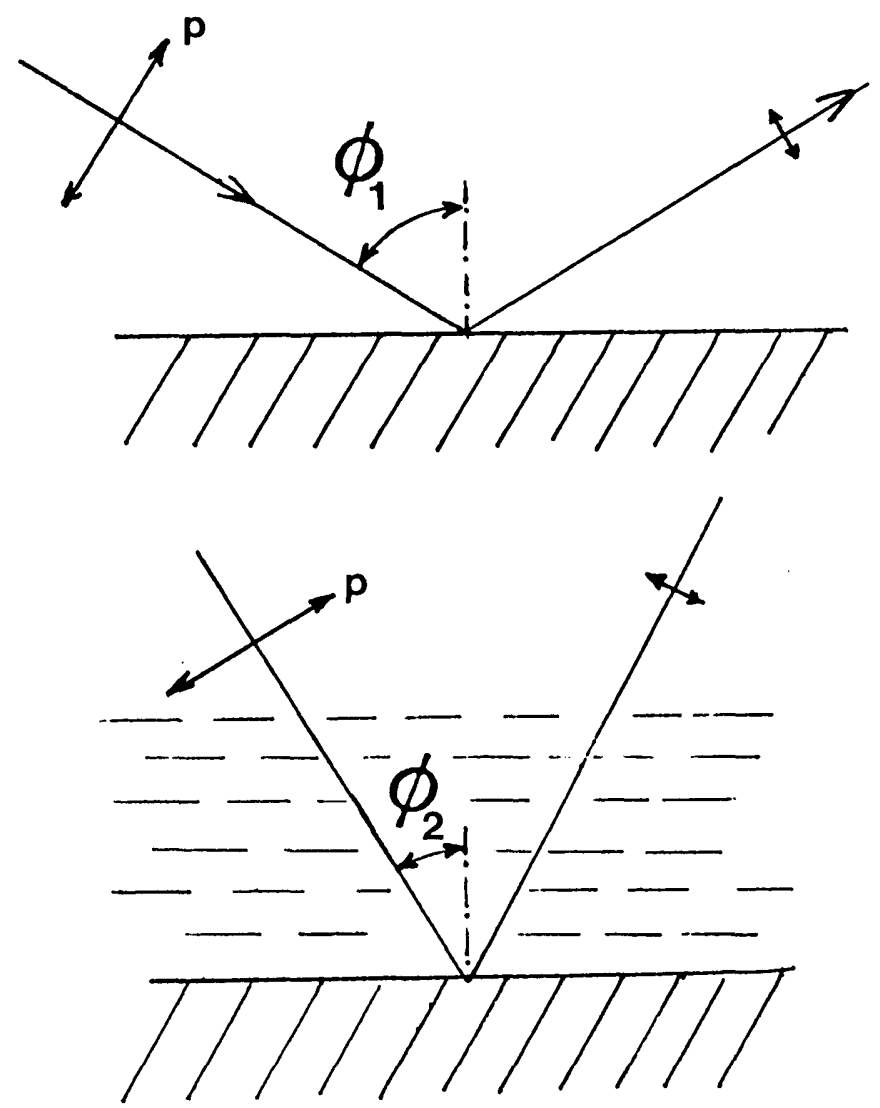

Fig. 1. Pseudo-Brewster angles $\phi_{1}$ (upper panel) and $\phi_{2}$ (lower panel) of minimum reflectance of $p$-polarized light are measured on the same surface of a bulk absorbing medium in two different media of incidence.
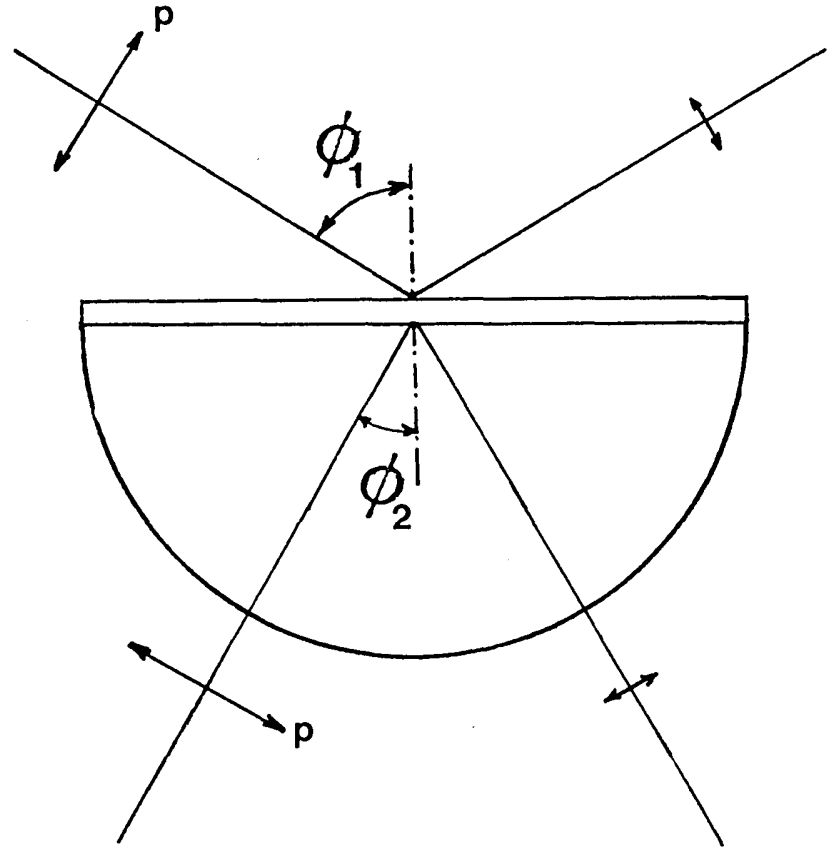

Fig. 2. Pseudo-Brewster angles $\phi_{1}$ and $\phi_{2}$ are measured in external and internal reflection, respectively, on an opaque film deposited upon a hemicylindrical or hemispherical transparent substrate.

two inert immersion liquids.) The situation is represented schematically in Fig. 1.

The preferred sample for the TAM is an opaque (optically thick) film with parallel-plane boundaries that is deposited (e.g., in vacuum) on a transparent substrate. The substrate takes the form of a hemicylinder or a hemisphere. $\phi_{1}$ and $\phi_{2}$ are measured in external and internal reflection, respectively, on the two parallel-plane faces of the film (Fig. 2). Ideally, $\phi_{1}$ should be measured in vacuum (before the sample is removed from the deposition chamber) or in an inert gas, to avoid the formation of a thin contamination or oxide layer on the film face in contact with the ambient. The substrate refractive index should be high so that $\phi_{1}$ and $\phi_{2}$ are sufficiently far apart.

\section{OPAQUE TIN FILM ON A ZnS SUBSTRATE: AN EXAMPLE}

As a specific example, we consider an opaque film of TiN deposited on a hemicylindrical Cleartran $(\mathrm{ZnS})$ substrate. The proposed method is tested by using the published values of the optical properties of TiN and ZnS (Refs. 8 and 9, respectively) and solving cubic Eq. (1) to determine the pseudo-Brewster angles $\phi_{1}$ and $\phi_{2}$ of external (in-air) and internal reflection, respectively, as functions of the wavelength of light $\lambda$ in the $0.5-1.5-\mu \mathrm{m}$ spectral range. The results thus obtained appear in Fig. 3 and simulate the actual data that one would obtain if the TAM were applied to the TiN-ZnS film-substrate system.

With the exact $\phi_{1}$ and $\phi_{2}$ derived from forward calculations, the known dielectric function of the transparent $\mathrm{ZnS}$ substrate, and working backwards using the analytical solution described in Section 2, we do obtain the same $\epsilon_{r}$ and $\epsilon_{i}$ of the opaque TiN film that we started with. This confirms the validity of the method. The spectra of $\epsilon_{r}$ and $\epsilon_{i}$, retrieved 


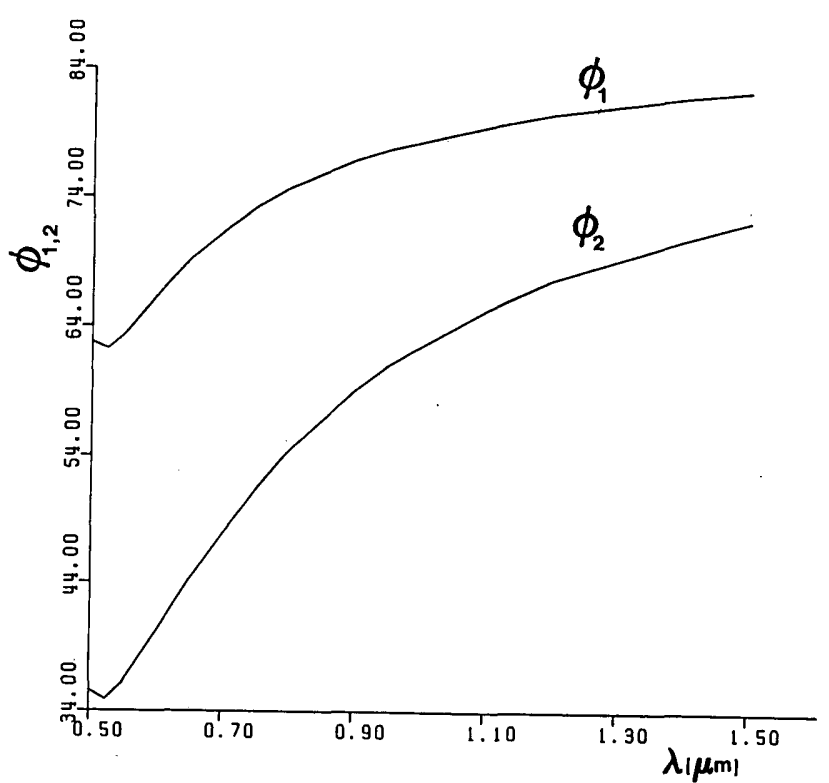

Fig. 3. Spectra of the pseudo-Brewster angles $\phi_{1}$ and $\phi_{2}$ of external (in-air) and internal reflection for a TiN film deposited on a Cleartran ( $\mathrm{ZnS})$ substrate. These curves are computed from Eq. (1) and the optical properties of TiN and $\mathrm{ZnS}$ given in Refs. 8 and 9, respectively.

from the pseudo-Brewster-angle spectra of Fig. 3, are shown in Fig. 4 by the continuous lines.

Because methods are available for the precise determination of the refractive index of a transparent substrate, ${ }^{10}$ the main source of error of the TAM is that associated with measurement of the angles of incidence of minimum parallel reflectance. To simulate the effect of angle-of-incidence errors, the exact pseudo-Brewster angles $\phi_{1}$ and $\phi_{2}$ are rounded off to the nearest $0.1^{\circ}$, which is equivalent to introducing a random error with a maximum possible value of $0.05^{\circ}$. Used in our analytical inversion method, these rounded-off $\phi_{1}$ and $\phi_{2}$ produce incorrect values of $\epsilon_{r}$ and $\epsilon_{i}$ that are represented by the crosses ( $X$ 's) and pluses ( + 's) in Fig. 4.

It is evident from Fig. 4 that the absolute errors $\left|\Delta \epsilon_{r}\right|$ and $\left|\Delta \epsilon_{i}\right|$ increase as $|\epsilon|$ itself increases, or, equivalently, as $\phi_{1}$ and $\phi_{2}$ increase well above $45^{\circ}$. This correlates well with the fact that, for large $\phi_{1}$ and $\phi_{2}$, the associated constant-pseudoBrewster-angle contours in the complex $\epsilon$ plane approximate semicircles centered on the origin, and the TAM loses its ability to resolve the argument of complex $\epsilon$. Only an estimate of $|\epsilon|$ can be obtained ( $\approx \tan ^{2} \phi_{1}$, in which $\phi_{1}$ is the pseudo-Brewster angle measured in air or vacuum). Table 1 gives numerical data corresponding to some of the points in Fig. 4.

Another potential source of error is that the incident light may not be exactly $p$ polarized. However, recent results ${ }^{11}$ indicate that the presence of a small $s$-polarized component in the incident light has no effect or a negligible effect on the position of the pseudo-Brewster angle, to first order.

\section{ERROR ANALYSIS}

In Section 4 we have considered the effect of errors of $\phi_{1}$ and $\phi_{2}$ on the determination of $\epsilon_{r}$ and $\epsilon_{i}$ by the TAM, with specific reference to a TiN film on a $\mathrm{ZnS}$ substrate (see Fig. 4 and Table 1). Because the TAM is analytical, it is possible to pursue an exact error analysis for this new method.

From Eq. (12) errors $\mathrm{d} \phi_{1}$ and $\mathrm{d} \phi_{2}$ cause a fractional error $\mathrm{d} x_{1} / x_{1}$ of $x_{1}\left[x_{1}=\epsilon_{r} / \epsilon_{01}\right.$ according to Eq. (7)], which is given by

$$
\begin{aligned}
\mathrm{d} x_{1} / x_{1}= & F_{1}\left(\mathrm{~d} a_{1} / a_{1}\right)+F_{2}\left(\mathrm{~d} b_{1} / b_{1}\right) \\
& +F_{3}\left(\mathrm{~d} a_{2} / a_{2}\right)+F_{4}\left(\mathrm{~d} b_{2} / b_{2}\right)
\end{aligned}
$$

in which

$$
\begin{aligned}
& F_{1}=\left[\epsilon_{0} a_{1} b_{2} /\left(\epsilon_{0} a_{1} b_{2}-a_{2} b_{1}\right)\right]-\left[a_{1} /\left(\epsilon_{0}^{2} a_{2}-a_{1}\right)\right], \\
& F_{2}=\left[2 b_{1} /\left(\epsilon_{0}^{3} b_{2}-b_{1}\right)\right]-\left[a_{2} b_{1} /\left(\epsilon_{0} a_{1} b_{2}-a_{2} b_{1}\right)\right], \\
& F_{3}=\left[\epsilon_{0}{ }^{2} a_{2} /\left(\epsilon_{0}^{2} a_{2}-a_{1}\right)\right]-\left[a_{2} b_{1} /\left(\epsilon_{0} a_{1} b_{2}-a_{2} b_{1}\right)\right], \\
& F_{4}=\left[\epsilon_{0} a_{1} b_{2} /\left(\epsilon_{0} a_{1} b_{2}-a_{2} b_{1}\right)\right]-\left[2 \epsilon_{0}{ }^{3} b_{2} /\left(\epsilon_{0}{ }^{3} b_{2}-b_{1}\right)\right] .
\end{aligned}
$$

Also, from Eqs. (4), an error of $\phi$ propagates into fractional errors of $a$ and $b$ given by

$$
\mathrm{d} a / a=A(\phi) \mathrm{d} \phi, \quad \mathrm{d} b / b=B(\phi) \mathrm{d} \phi,
$$

in which

$$
\begin{aligned}
& A(\phi)=\left(\cos ^{2} 2 \phi+4 \cos 2 \phi+7\right) /[\sin 2 \phi(1+0.5 \cos 2 \phi)] \\
& B(\phi)=(10+2 \cos 2 \phi) / \sin 2 \phi .
\end{aligned}
$$

Finally, from Eq. (5) an error $\mathrm{d} x_{1}$ of $x_{1}$ causes an error $\mathrm{d} y_{1}$ in the estimation of $y_{1}$ that is given by

$$
\mathrm{d} y_{1} / y_{1}=G\left(\mathrm{~d} x_{1} / x_{1}\right)
$$

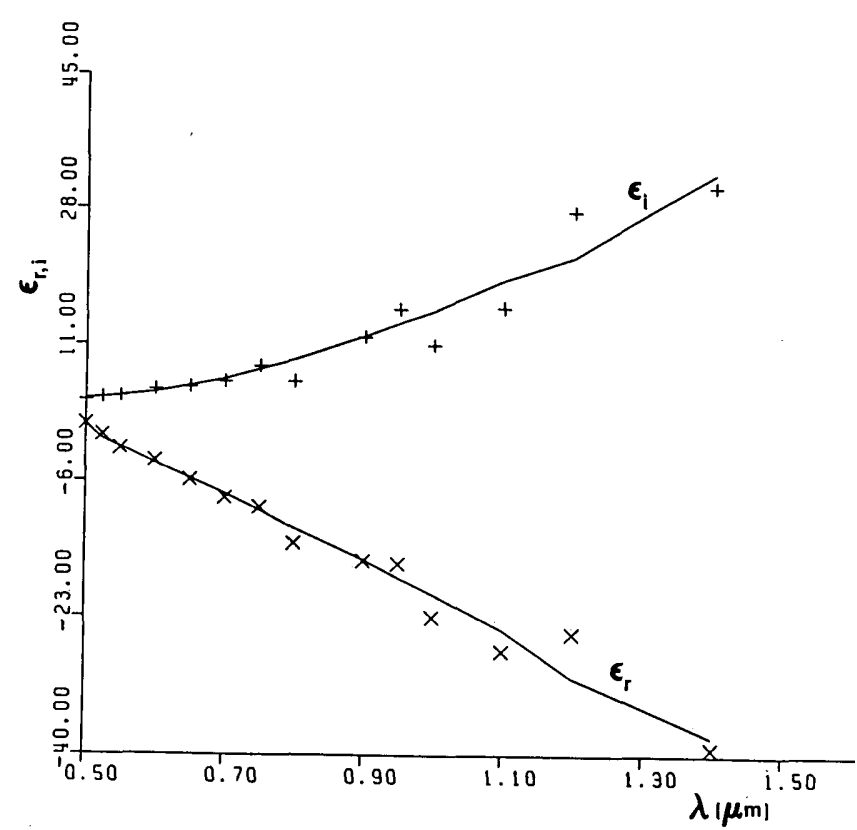

Fig. 4. Spectra of the real and imaginary parts, $\epsilon_{r}$ and $\epsilon_{i}$, of the complex dielectric function of TiN (continuous lines) obtained by applying the TAM to the simulated pseudo-Brewster-angle data of Fig. 3. The crosses (X's) and pluses (+'s) represent $\epsilon_{r}$ and $\epsilon_{i}$, respectively, as determined by the TAM when the exact pseudo-Brewster angles are rounded off to the nearest $0.1^{\circ}$. This procedure simulates the effect of angle-of-incidence errors. 
Table 1. Application of the TAM to the TiN/ZnS Film-Substrate System ${ }^{a}$

\begin{tabular}{crrrrrrr}
\hline$\lambda(\mu \mathrm{m})$ & $\epsilon_{r}(\mathrm{TiN})$ & $\epsilon_{i}(\mathrm{TiN})$ & $n(\mathrm{ZnS})$ & $\phi_{1}\left({ }^{\circ}\right)$ & $\phi_{2}\left({ }^{\circ}\right)$ & $\Delta \epsilon_{r}$ & $\Delta \epsilon_{i}$ \\
\hline 0.5 & 1.084 & 4.224 & 2.420 & 62.7945 & 35.6561 & 0.1171 \\
0.6 & -3.740 & 5.175 & 2.363 & 66.4406 & 40.1117 & -0.1673 & -0.0480 \\
0.7 & -7.475 & 6.890 & 2.332 & 71.3729 & 47.8874 & -0.1809 & -0.1553 \\
0.8 & -11.808 & 9.223 & 2.314 & 74.7611 & 54.4271 & -1.8779 & -2.7533 \\
0.9 & -15.875 & 12.296 & 2.301 & 76.9128 & 59.1369 & -0.1236 & -0.2195 \\
1.0 & -20.033 & 15.422 & 2.293 & 78.3832 & 62.5478 & -2.8927 & -4.4004 \\
\hline
\end{tabular}

${ }^{a} \lambda$ is the wavelength of light. $\epsilon_{r}(\mathrm{TiN})$ and $\epsilon_{i}(\mathrm{TiN})$ are real and imaginary parts of the complex dielectric function of the TiN film from Ref. 8 . $n(\mathrm{ZnS})$ is the refractive index of the ZnS substrate from Ref. $9 . \phi_{1}$ and $\phi_{2}$ are the pseudo-Brewster angles of external and internal reflection calculated from Eq. (1). $\Delta \epsilon_{r}$ and $\Delta \epsilon_{i}$ are the errors of $\epsilon_{r}$ and $\epsilon_{i}$ caused by rounding off $\phi_{1}$ and $\phi_{2}$ to the nearest $0.1^{\circ}$ in the TAM. It is apparent that the TAM is reasonably accurate in the visible range but not so accurate in the IR for this particular system.

in which

$$
\begin{aligned}
G & =-\left[x_{1}{ }^{2} X_{1}{ }^{3 / 2}+\left(b_{1} / 4\right) x_{1}\right] /\left[\left(a_{1}-x_{1}{ }^{2}\right) X_{1}{ }^{3 / 2}+X_{1}{ }^{2}\right], \\
X_{1} & =a_{1}{ }^{2}-b_{1} x_{1} .
\end{aligned}
$$

This completes the required error analysis for the TAM.

\section{DOMAIN OF APPLICABILITY OF THE TWO-ANGLE METHOD}

On the basis of the example given in Section 4 and others not present here, we can conclude that the TAM becomes inaccurate (or even unusable) when any one of the following conditions is met:

(1) Both $\phi_{1}$ and $\phi_{2}$ are $\gg 45^{\circ}$. This is the case of large $|\epsilon|$, e.g., of high-reflectance metals in the IR.

(2) $\left|\epsilon_{i} / \epsilon_{r}\right| \ll 1$; i.e., near an absorption edge or a plasma resonance. In this case $\epsilon_{i}$ is ill determined.

(3) A high-index-of-refraction substrate (or immersion medium) is not available, so that the difference $\phi_{1}-\phi_{2}$ is not sufficiently large (e.g., is $<5^{\circ}$ ).

The error analysis of Section 5 can be used as a guide for deciding when to use or not to use the TAM.

\section{CONCLUSION}

In this paper a new and simple method has been introduced for determining the complex dielectric function $\epsilon$, or complex refractive index $N=\epsilon^{1 / 2}$, of an opaque film or bulk substrate from two pseudo-Brewster angles measured in two different transparent media of incidence. This TAM has the following advantages:

(1) No photometric or polarimetric measurements are required.

(2) The optical properties are determined from the two measured angles analytically, explicitly, and noniteratively (see Section 2).

(3) The effect of angle-of-incidence errors on the calcu- lated optical properties is also analytically tractable (see Section 5).

Conditions under which the TAM may not be applicable have also been identified (see Section 6).

\section{ACKNOWLEDGMENTS}

This research was supported in part by the National Science Foundation and was presented at the Optical Interference Coatings Topical Meeting of the Optical Society of America, April 12-15, 1988, Tucson, Arizona. The assistance of A. ElSaba in performing the numerical calculations of Section 4 is also appreciated.

\section{REFERENCES}

1. See, e.g., D. L. Greenaway and G. Harbeke, Optical Properties and Band Structure of Semiconductors (Pergamon, New York, 1968).

2. See, e.g., H. A. Macleod, Thin-Film Optical Filters, 2nd ed. (Macmillan, New York, 1986).

3. See, e.g., W. R. Hunter, "Measurement of optical constants in the vacuum ultraviolet spectral region," in Handbook of Optical Constants of Solids, E. D. Palik, ed. (Academic, New York 1985).

4. See, e.g., D. E. Aspnes, in "The accurate determination of optical properties by ellipsometry," in Handbook of Optical Constants of Solids, E. D. Palik, ed. (Academic, New York, 1985).

5. S. P. F. Humphreys-Owen, "Comparison of reflection methods for measuring optical constants without polarimetric analysis, and proposal for new methods based on the Brewster angle," Proc. Phys. Soc. London 77, 949-957 (1961).

6. R. M. A. Azzam, "Maximum minimum reflectance of parallelpolarized light at interfaces between transparent and absorbing media," J. Opt. Soc. Am. 73, 959-962 (1983).

7. S. Y. Kim and K. Vedam, "Analytic solution of the pseudoBrewster angle," J. Opt. Soc. Am. A 3, 1772-1773 (1986).

8. C.-G. Ribbing and E. Valkonen, "Optical properties of noble metal like in films," Thin Film Technologies II, J. R. Jacobsson, ed., Proc. Soc. Photo-Opt. Instrum. Eng. 652, 166-178 (1986).

9. M. Debenham, "Refractive indices of zinc sulfide in the 0.40513- $\mu$ m wavelength range,". Appl. Opt. 23, 2238-2239 (1984).

10. See, e.g., Ref. 9 and papers cited therein.

11. R. M. A. Azzam and A. M. El-Saba, "Reflectance of an absorbing substrate for incident light of arbitrary polarization: appearance of a secondary maximum at oblique incidence," Appl. Opt. 27, 4034-4037 (1988). 